LA-UR- 09-0060'7y

Approved for public release:

distribution is unlimited.

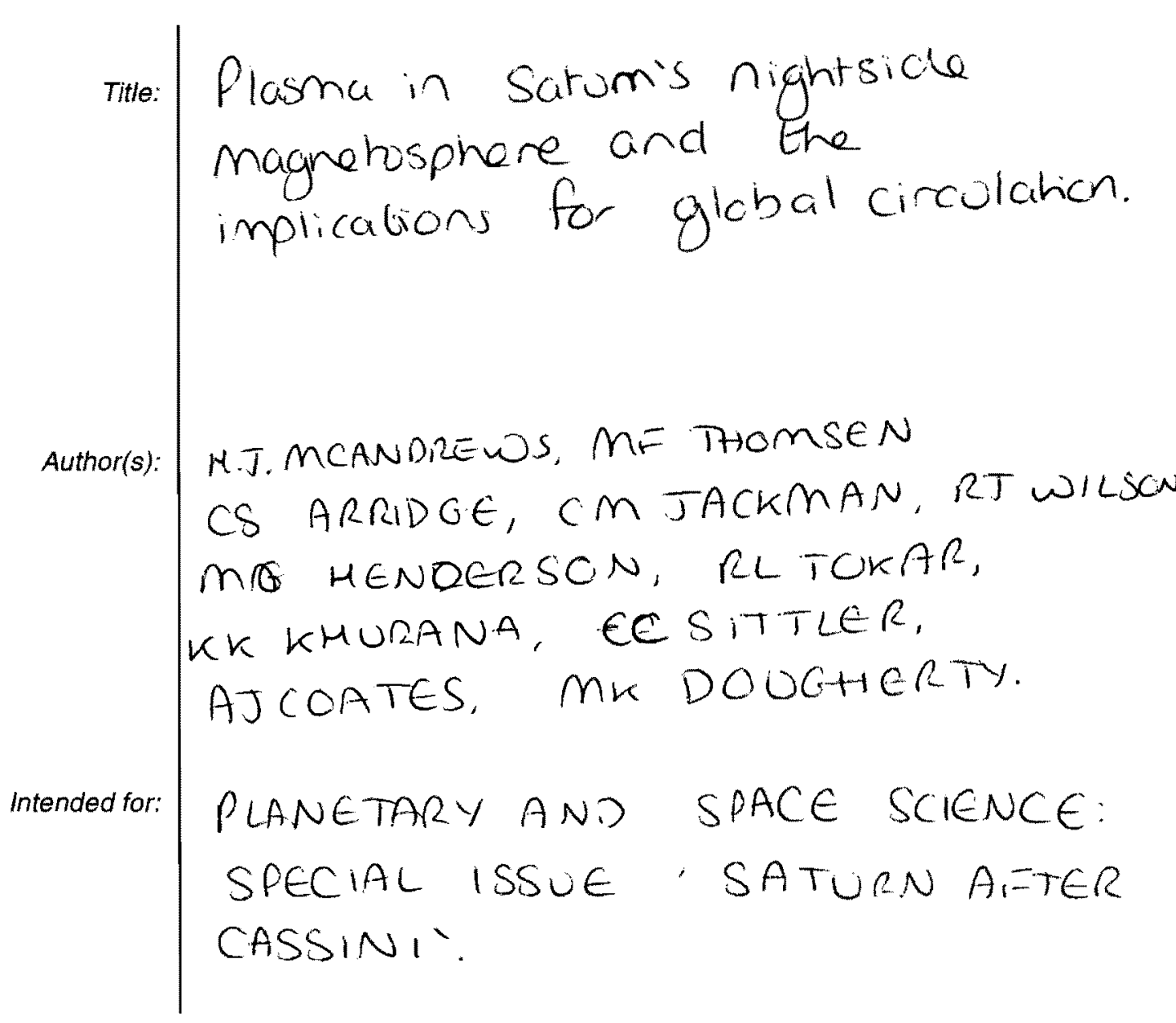

LosAlamos

NATIONAL LABORATORY

Los Alamo National Laboratory, an affirmative action/equal opportunity employer, is operated by the Los Alamo National Security, LLC for the National Nuclear Security Administration of the U.S. Department of Energy under contract DE-AC52-06NA25396. By acceptance
of this article, the publisher recognizes that the U.S. Government retains a nonexclusive, royalty-free license to publish or reproduce the published form of this contribution, or to allow others to do so, for U.S. Government purposes. Los Alamos National Laboratory requests that the publisher identify this article as work performed under the auspices of the U.S. Department of Energy. Los Alamos National endorse the viewpoint of a publication or guarantee its technical correctness.

Form $836(7 / 06)$ 


\section{Plasma in Saturn's nightside magnetosphere and the implications for}

\section{2 global circulation}

3 H. J. McAndrews", M. F. Thomsen', C. S. Arridge, C. M. Jackman', R. J. Wilson , M. G.

4 Henderson, R. L. Tokar , K.K. Khurana ${ }^{\text {h }}$ E. C. Sittler', A. J. Coates, M. K. Dougherty

6 "H. J. McAndrews", hazelm@lanl gov, Space Science and Applications, Los Alamos

7 National Laboratory, P.O. Box 1663, D466, Los Alamos, NM 87545, USA. Telephone

$8+15056670186 \mathrm{Fax}+15056657395$

9 "M. F. Thomsen, mthomsenalanl gov, Space Science and Applications, Los Alamos

10 National Laboratory, P.O. Box 1663, D466, Los Alamos, NM 87545, USA.

11 "C. S Arridge, csa amssl ucl ac.uk, Mullard Space Science Laboratory, Holmbury St

12 Mary, Dorking, Surrey, RH5 6NT, UK, and Centre for Planetary Sciences at

13 UCL/Birkbeck, University College London, Gower Street, London, WC1E 6BT, UK

$14{ }^{\mathrm{d} C . M .}$ Jackman, c.jackman@imperial ac.uk, Space and Atmospheric Physics Group,

15 Imperial College London, Prince Consort Road, SW7 2BW, UK.

16 "R. J. Wilson, rjwalanl.gov, Space Science and Applications, Los Alanos National

17 Laboratory, P.O. Box 1663, D466, Los Alamos, NM 87545, USA

18 'M. G. Henderson, mghenderson@lanl.gov, Space Science and Applications, Los

19 Alamos National Laboratory, P.O. Box 1663, D466, Los Alamos, NM 87545, USA.
28 Mary, Dorking. Surrey, RH5 6NT, UK and Centre for Planetary Sciences at

29 UCL/Birkbeck, University College London, Gower Street, London, WC1E 6BT, UK.

$30 \quad{ }^{k}$ M. K. Dougherty, m.dougherty@imperial.ac.uk, Space and Atmospheric Physics Group,

31 Imperial College London, Prince Consort Road, SW7 2BW, UK

\section{Abstract}

34 We present a bulk ion flow map from the nightside, equatorial region of Saturn's

35 magnetosphere derived from the Cassini CAPS ion mass spectrometer data. The map

36 clearly demonstrates the dominance of corotation flow over radial flow and suggests that

37 the flux tubes sampled are still closed and attached to the planet up to distances of $50 \mathrm{R}$

38 The plasma characteristics in the near-midnight region are described and indicate a 
Keywords: Saturn; magnetospheric; ions; planetary wind; magnetotail

\section{1. Introduction}

59 When considering the global dynamics of Saturn's magnetosphere, parallels are

60 immediately drawn with the magnetic systems of both Earth and Jupiter. At Earth, the

61 magnetosphere is forced into convective motion by reconnection with the interplanetary

62 field at the dayside magnetopause and the subsequent merging of the opened field lines in

63 the magnetospheric tail. The newly-closed field lines rotate back around to the dayside,

64 completing the so-called Dungey cycle (Dungey, 1961)

65 By contrast, despite evidence for interaction with the solar wind (Walker and Russell,

66 1985: Khurana, 2001 the fast rotation and significant internal mass-sources in Jupiter's

67 magnetosphere dominate the dynamics of the plasma and fields there. Vasyliunas (1983)

68 described a possible internally-driven scenario at Jupiter, in which plasma produced by

69 the moon lo continually loads the magnetic field lines. Due the interchange process this

70 plasma diffuses outward over many rotations. The filled flux tubes rotate around the dusk

71 magnetosphere where the reduction in solar wind confinement allows the flux tubes to

72 lengthen. Eventually, the magnetic stress can no longer confine the plasma and the field

73 lines reconnect or break open to release the plasma downtail as a series of plasmoids in

74 what has previously been termed the "planetary wind" (Michel and Sturrock, 1974). The

75 emptied flux tubes retum to the dayside, depleted of plasma, to complete this circulation 
91 reconnection events (Jackman et al., 2007, 2008; Hill et al., 2008). Only five such event

92 have been identified to date. In addition to these in-situ observations, remote sensing by

93 the ENA imager detected energetic neutral atoms originating from the tail region between

$94 \sim 20-30 R_{x}$ suggestive of local acceleration of ions associated with a substorm-type energisation (Mitchell et al. 2005). Furthermore one of these bursts was associated with the in-situ observation of a plasmoid (Hill et al., 2008). Such brightenings in the ENA signature are not uncommon (Carbary et al., 2008) which suggests that the low in-situ

detection rate is because Cassini has not been in a favorable viewing location during its excursions into the tail due largely to the warping of the current sheet out of the

equatorial plane (Arridge et al., 2008b).

Thus, there is a noteworthy amount of evidence for reconnection-related dynamics in the nightside magnetosphere. Furthermore, the evidence for mass-release in Saturn's

magnetosphere is unambiguous; there is no infinite build-up of mass in the system as demonstrated by the relatively stable mass profile in Arridge et al. (2007) which suggests a fairly constant loss rate and by the presence of depleted flux tubes, which contain remnants of inner magnetospheric material in the middle and outer magnetosphere. The principal motivation of the present study is to measure directly the characteristics of the large-scale plasma flows in the nightside magnetosphere to provide insight to the dynamical processes. Are radial flows observed on a large scale both outwards, suggestive of tailward plasma release, and inwards, indicating the return of newly closed field-lines? Does the relative density of plasma on these field lines support the idea of 2 plasma release? Can we find evidence of where the Vasyliunas and/or Dungey cycles are 


\section{2. Data set}

$1172 . /$ Instrumentation

118 Plasma parameters are derived from the Ion Mass Spectrometer (IMS) and the Electron

119 Spectrometer (ELS), which constitute part of the Cassini Plasma Spectrometer (CAPS)

120 (Young et al., 2004). The IMS provides energy and compositional information for ions

121 with an energy per charge (E/q) of $\mathrm{I} \mathrm{eV} / \mathrm{e}$ to $50 \mathrm{keV} / \mathrm{e}$ using an electrosiatic analyser and

122 a subsequent time-of-flight detector. The ELS is an electrostatic analyser with

123 measurement range of $0,6 \mathrm{eV} / \mathrm{e}$ to $28 \mathrm{keV} / \mathrm{e}$. The IMS and ELS fields of view are

124 constructed by sweeping the instrument collection apertures $\left(160^{\circ} \times 8.3^{\circ}\right.$ and $160^{\circ} \times 5^{\circ}$

125 for IMS and ELS, respectively) azimuthally by an actuating platform moving at $-1 \mathrm{~s}^{-1}$.

126 The maximum solid-angle coverage nomally allowed by the actuator is $\sim 2 \pi \mathrm{sr}$.

127 One-minute averaged magnetic field data were obtained from the Fluxgate Magnetometer

128 (FGM) (Dougherty et al., 2004).

129

$130 \quad 2.2$ Deriving plasma moments
131 Moments of the ion distribution (density, temperature and bulk flow velocities) are

132 determined by a process of forward modelling of the assumed convective anisotropic

133 Maxwellian distributions of two species with the same bulk flow velocity (Wilson et al.,

134 2008). The best fit is selected by employing a sequence of minimisation techniques

135 applied to a comparison of the observed and simulated count rate spectrum It is

136 necessary to pre-select the ion species to be modelled, and for the data shown here we

137 specified a light ion $\left(\mathrm{H}^{+}\right)$and a water group $\left(\mathrm{W}^{+}\right)$component based on lower-resolution

138 time-of-flight measurements of the IMS. Although we assume mass=17 amu for the

139 heavy ion species, the population may actually include $\mathrm{O}^{+}, \mathrm{OH}^{+}, \mathrm{H}_{2} \mathrm{O}^{+}$and $\mathrm{H}_{3} \mathrm{O}^{+}$iens, but

140 the derived moments are relatively insensitive to the actual composition. The calculation

141 is carried out over $14 \times 32$ second intervals, resulting in ion moments with a $\sim$ seven

142 minute resolution. Further details regarding the calculation can be found in Tokar et al.

$143(2006)$ and Wilson et al $(2008)$

144 The electron moments are derived by integrating the particle distribution over three-

145 dimensional velocity space. In general, the electrons have a high thermal speed relative

146 to their bulk speed and so, in the absence of anisotropic pitch-angle distributions, do not

147 have significant directionality. Therefore, the electrons sampled in one direction can

148 generally be considered representative of the local population and in the momen 


\section{3, Observations}

158

164 aperture through the peak of the ion flow, which is strongly directional in the spacecratt

165 frame since the flow speed is comparable to or exceeds the thermal speed. The

plots in a spacecraft-centered frame at the bottom of the figure. The fields of view for the periods corresponding to the red bars on the spectrogram are shown. Between $0900-$

0915 UT the detector is sweeping through the direction of strictly azimuthal flow

(triangle) and counts are detected allowing us to derive the actual flow direction (upsidedown triangle). Note that the actual flow direction is very close to the direction expected

for rigidly corotating plasma and indicates the lack of any significant radial flow at this

time In the second case $(0915-0930$ UT), the actuator does not sweep the instrument

through the flow direction and the counts disappear.

The energy structure of the ion counts in the top panel is due to the presence of two main

ion populations, a light (primarily $\mathrm{H}^{+}$) and a heavy (primarily water-group, denoted $\mathrm{W}^{+}$)

component, which for the same common flow velocity have energy-per-charge

proportional to the ion mass (c.f. Young et al., 2005). In extracting moments these populations must be successfully identified and separated.

These data demonstrate the characteristics of plasma in the outer magnetosphere and

highlight the sparse nature of the ions detectable by the IMS. At the start of the interval in

Figure 1, there are no ion counts due to the instrument not sampling the flow direction

The electron counts are low and have energies $-50-500 \mathrm{eV}$ (the intense band of electrons

at the lowest energies is due to spacecraft photoelectrons trapped in the potential well surrounding the spacecraft), At -0900 UT the ions appear as the spacecraft reorients. 
$203 \quad 3.2$ Tail survey

204 Figure 2 shows the low-latitude passes from the Cassini orbits from 12 October 2005 to magnetosphere at a range of planetocentric distances ( 9 to $50 \mathrm{R}_{s}$ ) and magnetic local times $(2000-0600 \mathrm{MLT})$

Intervals to include in the survey were selected based on the criterion that there were sufficient counts on which to perform the ion moments analysis. Since the plasma sheet/magnetodisc is 'pushed' northward out of the equatorial plane, most encounters with it have been found near $10-13^{\circ}$ in latitude at $\sim 40 R_{s}$. Because the plasma sheet also exhibits rotational modulation consistent with the planetary spin period (Arridge et al.

$2008 \mathrm{~b}$ ) and may flap up and down in response to variations in the solar wind, entries into the plasma sheet are, in practice, sporadic

The bulk flow of the ions can only be measured if the velocity vector lies within the instrument field of view as demonstrated in Figure 1. Optimal periods are where the flow is close to the centre of the anode fan and the actuation sweep so that the detector samples the full beam. In total, 35 days between 12 October 2005 and 24 May 2007. containing plasma sheet encounters where the IMS was able to sample the peak of the ion flow were collated, providing $\cdots 44$ hours of ion data for use in the tail survey

In Figure 3 the derived flow vectors are drawn originating from the spacecraft position at the time of measurement and projected onto the equatorial plane. The vectors are 
that the flows in this region are still predominately in the corotational direction, even at large distances from the planet $\left(\sim 50 \mathrm{R}_{\mathrm{s}}\right)$

The velocity vectors in Figure 3 indicate significantly lower flow speeds than the local corotational speed (compare with the scale arrows in Figure 3) at distances from $\sim 15 R_{s}$ outwards. The general flow pattern illustrates the departure from purely azimuthal flow outside of $\sim 20 R_{s}$ in the post midnight sector, where the flow appears to be more dawnward. In this region the flows would not appear likely to circulate on around to the dayside region without a substantial sunward acceleration. There are departures from the purely azimuthal flow with clear instances of more radial flow in agreement with results from Voyager (Lazarus and McNutt, 1983). However, no instances of rapid radial flow. akin to the plasmoid signatures of Hill et al. (2008) (which have been included on the flow pattern for comparison) are observed. Instead, the radial flow is superimposed on the azimuthal flow as a gradual motion of the bulk plasma. There is no notable correlation of density with outwardly-moving flows.

Figure 4 shows several of the derived plasma properties as a function of radial distance from Saturn. In the top panel, the measured azimuthal velocity is plotted and compared with the profile for strictly corotating flow (dashed line). The second panel contains the angle between the direction of bulk and azimuthal flows for each radial distance sampled, with a line at zero marking the transition between inward and outward flow. In the third panel are the proton (green) and water group (red) densities together with the $\mathrm{W}^{+} / \mathrm{H}^{+}$ densify ratio, and the bottom panel contains the parallel temperatures for the light (green) and heavy (red) ions

It is clear that the data in all panels of Figure 4 show a marked change in the nature of the plasma and flows beyond $\sim 15-20 \mathrm{R}_{s}$. Outside of $15 \mathrm{R}_{s}$ the azimuthal velocity no longer increases with radial distance and remains approximately constant at $\sim 100-200 \mathrm{kms}^{-1}$ out to $46 R_{s}$. The azimuthal flow velocities outside of $-25 R_{5}$ are in accord with those derived from the MIMI-INCA data (Kane et al., 2008). Azimuthal velocities as low as $0.25 \mathrm{~V}_{\mathrm{CR}}$ highlight the significant mass-loading on these field lines in agreement with earlier findings (Frank et al., 1980; Richardson, 1986). The subcorotation observed also is in qualitative agreement with the lagging field observed in this region (Arridge et al., 2006).

In general, inward bulk flows are only observed inside of $\sim 15 R_{s}$ as demonstrated by the negative angles plotted in the second panel of Figure 4 inside of this distance. These inward (and outward) motions are most likely the result of global magnetospheric motion

Outside of $17 \mathrm{R} s$, only outward motion is observed, and the flow angle increases with radial distance resulting in more tailward flows (and less strictly azimuthal flows) with increased distance from the planet. Indeed, an examination of the flow pattern in Figure 3 
sunward and return to the dayside

Inside of $15 R_{S}$ there is a steep drop-off with distance in the densities of both the water group and light ions, although the decline of the density ratio signifies a sharper decrease

in the water group ions relative to the light ions. This drop-off is as expected for a dipole

field. Outside of $15 \mathrm{Rs}_{\mathrm{s}}$ the densities of both species decrease comparably with increasing

radial distance as evidenced by the flat profile of the density ratio until $\sim 35 R_{s}$ when

there is a slight increase in the density ratio.

Inside of $15 \mathrm{R}_{S}$ the parallel ion temperatures (Figure 4, third panel) increase with radial

distance due to the scattering of the pick-up ion population from the inner magnetosphere

(e.g. Wilson et al, 2008). Once the transition at $\sim 15 \mathrm{R}_{\varsigma}$ is crossed, however, they show

an almost constant or slowly declining profile.

In the final panel we plot the Alfven travel time to the planet using the local field strength

and proton density. The proton density is used since it is representative of the density

long the field line. Values start at sub-one hour increasing to a maximum of four hours

at $>40 \mathrm{Rs}_{\mathrm{s}}$

One of the most striking aspects of the ion flow pattern and associated parameters is the

lack of inwardly moving flux tubes in the outer magnetosphere. Such motion would be

observed if field lines that had recently undergone mass-release were returning to the

inner magnetosphere depleted and, due to the reduction of plasma-loading, moving at corotational or possibly even super-corotational speeds. Furthermore, the planetary wind model would favor the release of the most equatorially confined, heavy ions; hence a pos mass-release flux tube should show a preferential depletion of water group ions. The remaining ions would likely have been energised in the magnetic reconnection that produced the mass release. Hence, one might expect the signature of such returning depleted flux tubes to be low density, energised, water-depleted ions distributions moving inwards and with faster azimuthal velocities than in the ambient plasma

No such ion signature is apparent in the intervals sampled. For example, the relatively flat density ratio profile in the third panel of Figure 4 suggests that no significant water group

depletion relative to the light ions is detected. The possible reasons for this are outlined in section 3.3

\subsection{Why are return flows not observed in the ion data?}

The lack of signatures of returning, depleted flux tubes in the intervals represented by Figures 3 and 4 could arise if a) there is no such inward flow in the region sampled, b) the

inflow channels are physically much narrower in azimuth than the outflow channels

(Chen and Hill, 2008), which makes them less likely to be included in this dataset, c) the $\mathrm{MS}$ is never orientated such that it is looking outward to detect inward flows or d) the 
300 low density, energised, post-reconnection ions have fluxes too low to be detectable by the

301 IMS. The look direction plots in Figure 1 demonstrate the situation in case c), where the

302 bottom left shows an interval with nearly complete viewing of the sky, but the bottom

303 right plot shows the pointing for an interval only sampling a portion of the sky and where

304 inflows could be missed.

305 In the case of $\mathrm{c}$ ), we can establish whether instrument pointing introduces a bias into the

306 results by comparing how often the IMS is sampling each direction and the percentage of

307 inward or outward flows detected. In Table 1 are the durations of the look and flow

308 directions observed for inward and outward flows outside of $22 R_{S}$. In this region of the

309 outer magnetosphere, according to Figure 3, almost no inward flows are observed. The

310 percentage of inward flows detected relative to the time the instrument is looking

311 outward is very small and in fact can be attributed to one single data point. Upon further

312 inspection there is no noticeable difference between this data point and the surrounding

313 ones and it may be a spurious result. Nevertheless it is clear that the IMS has plenty of

314 opportunity to sample inward flows (it is looking outward for seven hours of the total

315 time), yet none are seen. Conversely, when the instrument looks inward, an abundance of

316 outward flows are detected.

317 In the case of d) we employ the ELS data, with its greater detection sensitivity, to

318 characterise the electrons in the regions sampled. This will establish whether the IMS is 
338 signatures of the mass-release processes, then it occurs across the whole region sampled

339 and not preferentially in the dusk magnetosphere as proposed by Vasyliunas (1983) and

340 Cowley et al. (2004).

341 Ion densities of two of the plasmoid events identified in Jackman et al. (2007) and Hill et

342 al. (2008) (March 42006 and August 4 2006) are also included in Figure 5 (squares). The

343 plasmoid densities for the first case have a range of values, extending to very low values

344 at the very limit of what can be observed in the IMS. For the second case, the densities

345 are near the top of the maximum values detected in this region. Therefore, were Cassini

346 in a favorable position on the dates included in this survey, to detect passing plasmoids,

347 we would expect to detect them. Positive identification of the plasmoids in the studies of

348 Jackman et al. $(2007,2008)$ and Hill et al. (2008) was achieved using magnetic field data.

349 A sharp, northward-turning of the primarily southward-pointing planetary field indicates

350 that the field lines have moved from a stretched- to a plasmoid-type configuration, yet no

351 such magnetic signatures have been associated with any of the intervals in this study,

352 which all appear to be on closed field lines. Furthermore, during none of the events

353 studied here is there evidence for a large rotation of the velocity from a largely azimuthal

354 flow direction to a strongly tailward flow, also indicative of plasmoid release (Hill et al.,

355 2008), and thus we conclude that despite similarities in density, the events included in 
375 of magnetic flux to the inner magnetosphere required due to flux conservation. As

376 described in section 3.3 there are a number of selection criteria that mitigate against

377 observing such features, namely the small scale-size of the inflow channels (Chen and

378 Hill, 2008), the low density expected in returning flux tubes and the likelihood of

379 favourable instrument viewing.

380 The flow pattern indicates that the observed flux tubes are likely to encounter the dawn

381 magnetopause, as the strongly dawnward flow observed implies, however, measurements

382 adjacent to the dawn magnetopause do not indicate a large build-up of plasma. Thus the

383 plasma either manages to rotate around dawn to the dayside, or it is lost either downtail

384 into interplanetary space.

385 The striking change in the characteristics of the plasma sheet beyond $\sim 15-20 \mathrm{R}_{\mathrm{S}}$ is most

386 likely due to the transition from the region dominated by closed flux tubes which are able

387 to complete an entire rotation of the planet (closed drift path), and the outer

388 magnetospheric flux tubes which may not remain closed as they convect through the tail

389 due to the centrifugal stresses brought on by significant mass loading. We also note that

390 this is just inside the expected hinging distance of the magnetotail current sheet (Arridge

391 et al., 2008b) and where the field lines start to become significantly stretched.

392 The Alfvén travel times in Figure 4 are shown to increase with radial distance. Travel

393 times of a few hours are consistent with a slight delay in the communication between the 
412 transport process (Hill and Dessler, 1976), and is $\eta \equiv \int(n(s) / B(s)) d s$, where the integral

413 is taken along a field line. We can approximate this integral as $\eta=\int n(z(s)) / B(z(s)) d s$,

414 where $n(z(s))=n_{0} \exp (-W / k T), n_{0}$ is the equatorial density and $T$ is the parallel ion

415 temperature (both derived from the IMS data), $W$ is the centrifugal potential and

$416 B(z(s))$ corresponds to the field strength derived from the magnetospheric field model of

417 Khurana et al. (2006). The centrifugal potential is derived using the assumption that a

418 single field line rotates with the same level of subcorotation as measured at the equator.

419 Since we derive equatorial azimuthal velocities we can work out the fraction of

420 subcorotation, $f$ and use $W=0.5 m f^{2} r^{2} \Omega^{2}$. The distance along the field line, $d s$ is

421 derived assuming a parabolic shaped field line in the stretched field region and we

422 multiply the values by the mass in $\mathrm{kg}$ to get the flux tube mass content plotted in the top

423 panel of Figure 6 (diamonds). There is significant spread in the data at all values of R,

424 indicative of the varying flux tube volumes encountered in the tail, presumably due to the

425 cycle of mass-release and loading. The values show a slight decrease with radial distance,

426 in particular the upper limit of the distribution of points declines with $\mathrm{R}$. This suggests

427 that despite the requirement that under no mass-loss the flux tube content is preserved,

428 there is a loss of flux tubes with a high mass content (at the upper limit of the profile)

429 with increasing $\mathrm{R}$. Thus, those are lost downtail and what we are effectively seeing are 
451 As we have shown, heavily loaded, stretched flux tubes may undergo mass-release in the

452 tail if some threshold relating to the mass-loading rate and field strength is reached.

453 However, it is possible that an alternative trigger may initiate the pinch-off process. For

454 example, Bunce et al. (2005) described an energetic particle event observed in the

455 magnetotail during SOI and attributed it to tail reconnection instigated by magnetospheric

456 compression by a solar wind corotating interaction region (CIR). Russell et al. (2008)

457 found that four of the five substorm events identified in Jackman et al. (2008) were

458 triggered when Titan was within one Saturn rotation of the highly-stretched tail field lines

459 at midnight local time.

460 We have not considered the effects of the external solar wind conditions or the position

461 of Titan during the events studied here, however we propose that in cases of fast rotation,

462 coupled with sufficient mass build-up, field lines can reconnect across the stretched

463 current sheet and drive plasmoid formation without, necessarily, an external driver. This

464 agrees with the proposal by Hill et al. (1974) and more recent work by Russell et al.

465 (2008) that state that due to the inefficiency of the reconnection process at Saturn's

466 dayside magnetosphere, accumulation of mass in the tail flux tubes may dominate

467 plasmoid formation, not closure of open flux generated at the dayside magnetopause. 
487 distances and no clear local time dependency in the nightside is found agreeing with

488 Chen and Hill, (2008).

489

490 Acknowledgements: The work at Los Alamos was performed under the auspices of the

491 US DOE and was supported by the NASA Cassini program. Work at Southwest Research

492 Institute was supported by JPL contract 1243218. Cassini is managed by the Jet

493 Propulsion Laboratory for NASA. Work at Imperial was supported by STFC. C.S.A. and

494 A.J.C. were supported in this work by the STFC rolling grant to MSSL/UCL. Part of this

495 work was discussed during a team meeting at the International Space Science Institute in

496 Bern, Switzerland. HJM, MFT, CSA and ECS acknowledge funding from ISSI to attend

497 this meeting.

498 References

500 Arridge, C. S., Achilleos, N., Dougherty, M. K., Khurana, K. K., Russell, C. T.,

501 Southwood, D. J., Cassini MAG Team, 2006. The Configuration of Saturn's

502 Magnetosphere as Observed by the Cassini Magnetometer, Eos Trans. AGU, 87(36),

503 Jt. Assem. Suppl., Abstract P43B-06

504

505 Arridge, C. S., Russell, C. T., Khurana, K. K., Achilleos, N., André, N. ,Rymer, A. M., 506 Dougherty, M. K., Coates, A. J., 2007. Mass of Saturn's magnetodisc: Cassini

507 observations, Geophys. Res. Lett., 34, L09108, doi:10.1029/2006GL028921. 
Dougherty M. K., Khurana, K. K., Neubauer, F. M., Russell, C. T., Saur, J., Leisner, J.

S., Burton, M. E., 2006. Identification of a dynamic atmosphere at Enceladus with the Cassini Magnetometer, Science, 311, 5766, 1406-1409.

Dungey J. W., 1961. Interplanetary magnetic field and the auroral zones, Phys. Rev.

$$
\text { Lett., 6, 48-49. }
$$

Eviatar, A., and Richardson, J. D., 1986. Corotation of the Kronian Magnetosphere, J.

Frank L. A., Burek, B. G., Ackerson, K. L., Wolfe, J. H., Mihalo, J. D., 1980. Plasma's in

Goertz C. K., 1983. Detached plasma in Saturn's front side magnetosphere, Geophys.

Hill T. W., 1979. Inertial limit on corotation, J. Geophys. Res., 83, A11, 6554-6558.

Hill, T. W., Dessler, A. J., and Michel, F. C., 1974. Configuration of the Jovian magnetosphere, Geophys. Res. Lett., $1,3$.

Hill T. W. and Dessler, A. J., 1976. Longitudinal asymmetry of the Jovian magnetosphere

564 Hill T. W., Thomsen, M. F., Henderson, M. G., Tokar, R. L., Coates, A. J., McAndrews, and the periodic escape of energetic particles, J. Geophys. Res., 81, 19, 3383-3386. 
600 Mitchell D. G., Brandt, P. C., Roelof, E. C., Dandouras, J.,. Krimigis, S. M., Mauk, B.

601 H., Paranicas, C. P., Krupp, N., Hamilton, D. C., Kurth, W. S., Zarka, P.,

602 Dougherty, M. K., Bunce, E. J., Shemansky, D. E., 2005. Energetic ion acceleration in

603 Saturn's magnetotail: Substorms at Saturn? Geo. Phys. Res., 32, L20S01.

604

605 Richardson, J.D., 1986. Thermal Ions at Saturn: Plasma Parameters and Implications, J.

606 Geophys. Res., 91, 1381.

607

608

Russell C. T., Jackman, C. M., Wei, H. Y., Bertucci, C., Dougherty, M. K., 2008. Titan's

Sittler E. C., Johnson, R. E., Jurac, S., Richardson, J. D., McGrath, M., Crary, F., Young, influence on Saturnian substorm occurrence, Geophys. Res. Lett., 35, L12105. D. T., Nordholt, J. E., 2004. Pickup ions at Dione and Enceladus: Cassini Plasma Spectrometer simulations, J. Geophys. Res., 109, A01214.

Tokar, R. L., Johnson, R. E., Hill, T. W., Pontius, D. H., Kurth, W. S., Crary, F. J., Young, D. T., Thomsen, M. F., Reisenfeld, D. B., Coates, A. J., Lewis, G. R., Sittler,

Vasyliunas V. M., 1983. Plasma distribution and flow. In Dessler A. J., (Ed), Physics of the Jovian magnetosphere. Cambridge planetary science series.

Walker R. J. and Russell, C. T., 1985. Flux transfer events at the Jovian magnetopause, J. Geophys. Res., 90, A8, 7397-7404.

Wilson R. J., Tokar, R. L., Henderson, M. G., Hill, T. W., Thomsen, M. F., Pontius Jr., D. H., 2008. Cassini Plasma Spectrometer Thermal Ion Measurements in Saturn's Inner Magnetosphere, J. Geophys. Res., 113, A12218, doi:10.1029/2008JA013486. 
Table 1: Instrument look directions

637 The inward and outward look durations are calculated from the percentage of time within

638 one sampling period ( $14 \times 32$ seconds) that the instrument is looking inward or outward.

639 The sum of these percentages is greater than $100 \%$ because the instrument can often see

640 both inward and outward flow directions within a sampling period. For this calculation

641 we make the assumption that the radial flow component from the moment calculations

642 within that sampling period is constant within a sampling interval, which could smooth

643 out short duration radial motion. Only periods outside of $22 \mathrm{R}_{\mathrm{S}}$ have been considered.

The top and middle panels contain time-energy spectrograms from the IMS and ELS instruments on July 32006 . Cassini is at $\sim 27 \mathrm{R}_{\mathrm{S}}$ and at a magnetic local time (LT) of 2150. The colour scale indicates the count rate; note the different scales for the ions and electrons. The ion spectrogram highlights the low counts and sporadic coverage due to pointing and limited entries into the plasmasheet typical of the tail orbits. The third panel shows the electron density together with the density threshold of $3 \times 10^{3} \mathrm{~m}^{-3}$. Below this are field of view plots for the IMS and ELS during the 15 minute intervals indicated by the red bars. The field of view plots are in a spacecraft-centered frame (OAS). The viewing for each polar anode $(1-8)$ is projected onto the sky (note that anode 2 is not displayed on the right-hand figure for clarity). A line from the observer to the centre of the plot is in the direction of positive $\mathrm{S}$, (the Cassini to Saturn direction), the north-south direction $(\mathrm{O})=\mathrm{S} \times($ Omega $\times \mathrm{S})$ where Omega is Saturn's spin axis and A completes the right-handed system. The azimuthal angle is shown on the outside of the outer circle and is about $S$, referenced to zero in the $O$ direction. The latitudinal angle is relative to $S$ where the inner dashed circle is $90^{\circ}$ latitude from $\mathrm{S}$. The directions from which strictly corotational and observed flows originate are indicated by a triangle and an upside-down triangle respectively. Between 0915 UT and 0930 UT the instrument was not looking into the flow direction, hence the bulk flow vector could not be determined.

Figure 2: Trajectory plot

Trajectory coverage plot for period covered in this study. The blue points denote the orbits surveyed in the ion data. The red points are where both ion and electron data were available. Only electron moments outside of $10 \mathrm{R}_{\mathrm{S}}$ and in the nightside between May 10 2006 to August 282006 were included in the analysis.

Figure 3: Equatorial flow pattern of ions

The velocity vector of each position was determined using the spacecraft position as the arrow's origin. Arrow length represents velocity and colour the total ion density. Scale arrows for strict corotation at radial distances of $5,15,25,35$ and $45 \mathrm{R}_{\mathrm{S}}$ have been indicated along with the magnitude of the corotation speed at that distance. The velocities are in a Saturn-centered equatorial system with the Sun toward the right of the figure. A sample magnetopause surface from Arridge et al. (2006) has been included for context. The flows detected during two plasmoid events on March 42006 and August 42006 (Hill et al., 2008) have been included. Note that the flow speed observed on March 4 681 
Duration of inward and outward flow detection beyond $22 R_{S}$

\begin{tabular}{|l|l|l|l|}
\hline $\begin{array}{l}\text { Instrument look } \\
\text { (plasma flow) } \\
\text { direction }\end{array}$ & $\begin{array}{l}\text { Look time (of 13 } \\
\text { hours in total) }\end{array}$ & $\begin{array}{l}\text { Duration of } \\
\text { observed flows }\end{array}$ & $\begin{array}{l}\text { Duration of flows } \\
\text { seen as a percentage } \\
\text { of opportunities }\end{array}$ \\
\hline Outward (inward) & 7 hours & 0.2 hours & $0.02 \%$ \\
\hline Inward (outward) & 13 hours & 12.8 hours & $98.0 \%$ \\
\hline
\end{tabular}


July 32006 (DOY 184)

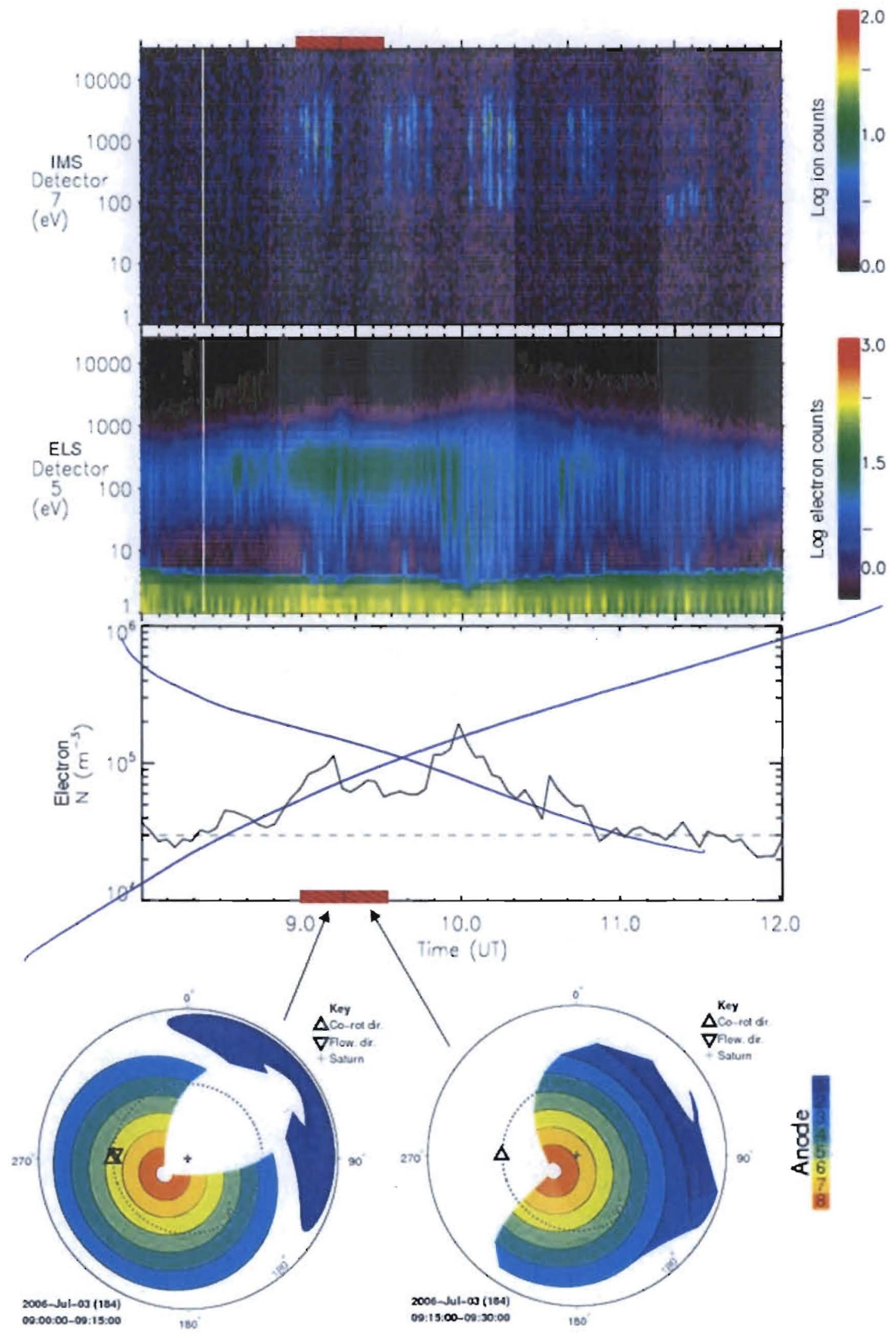




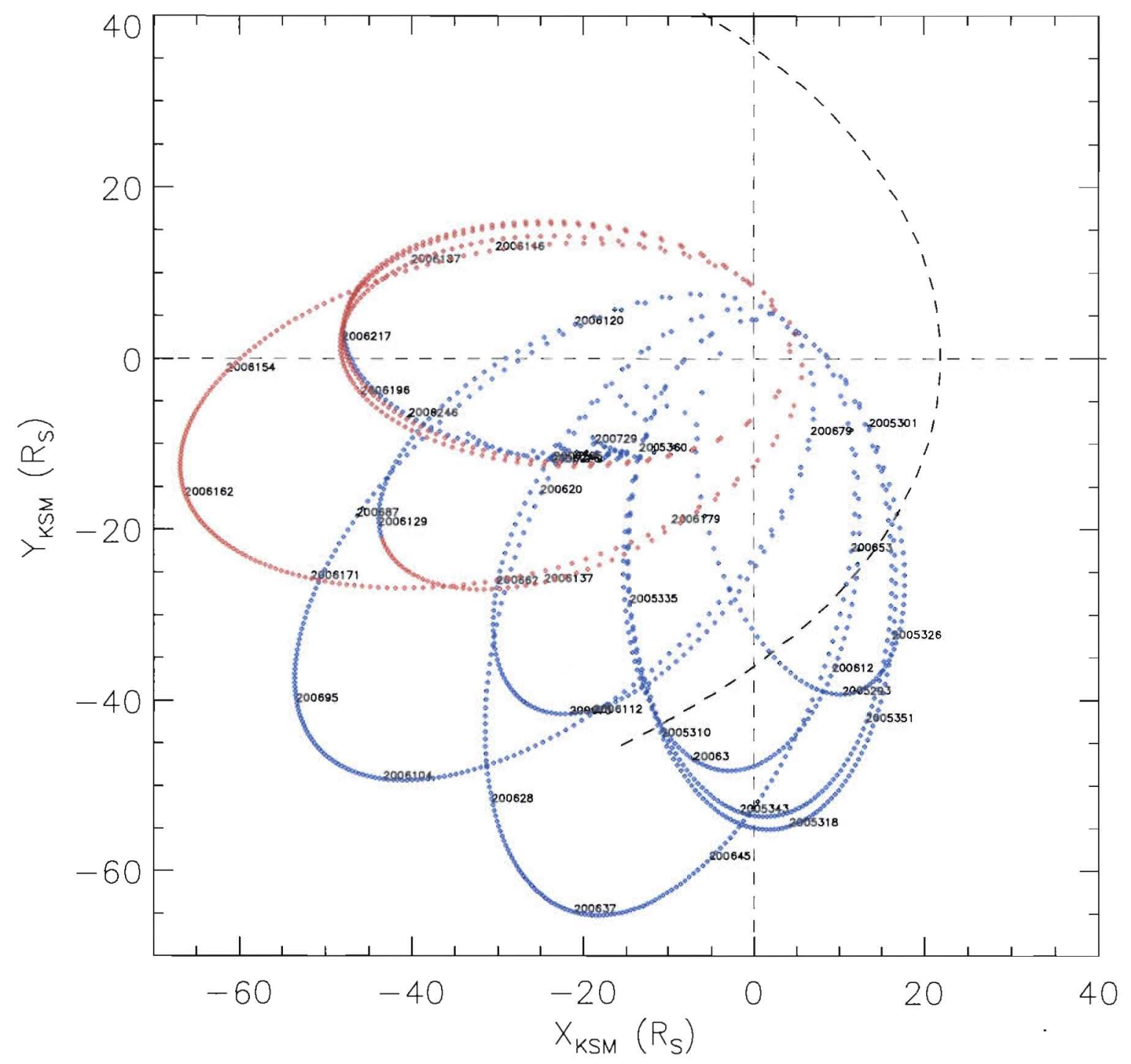




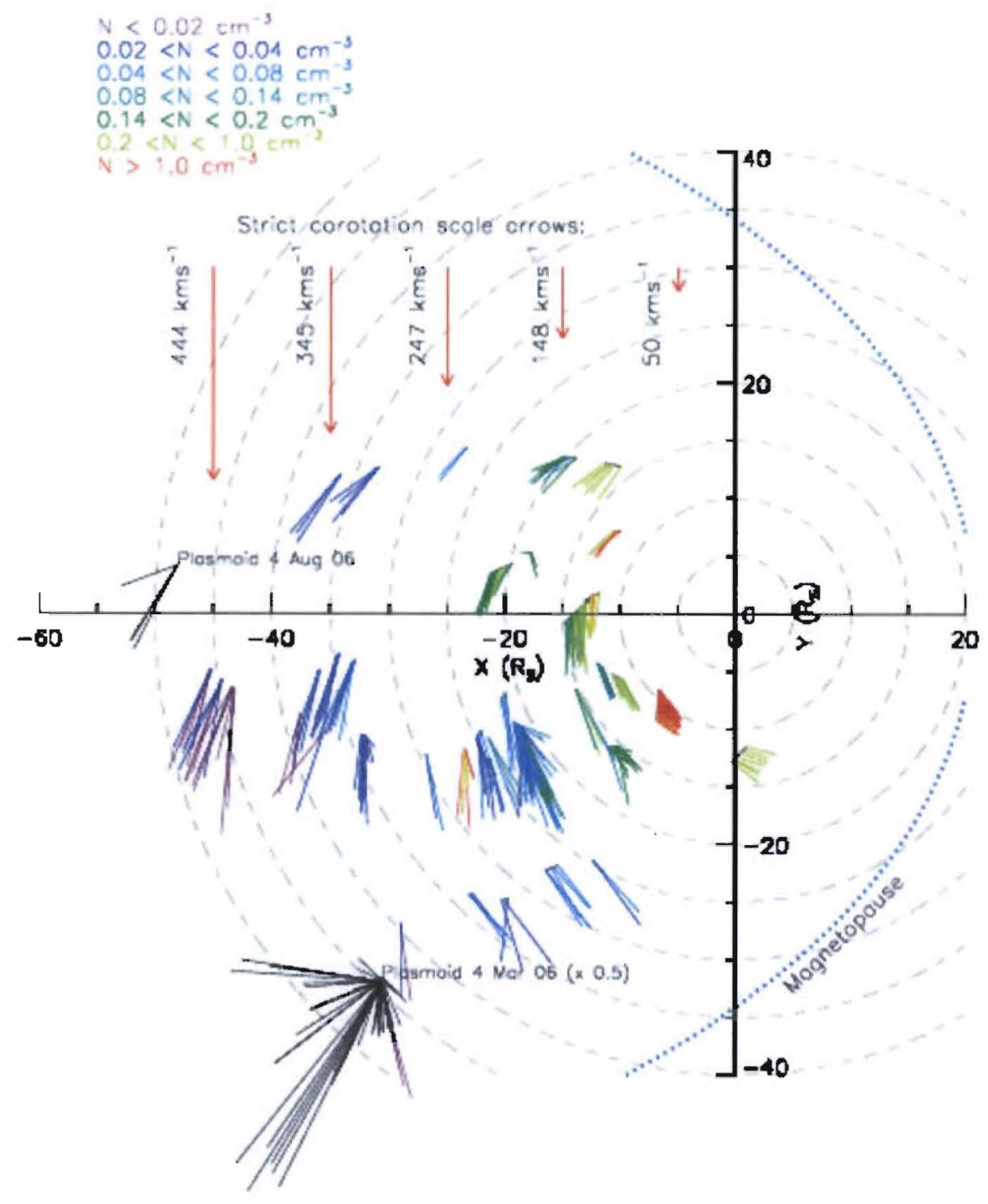




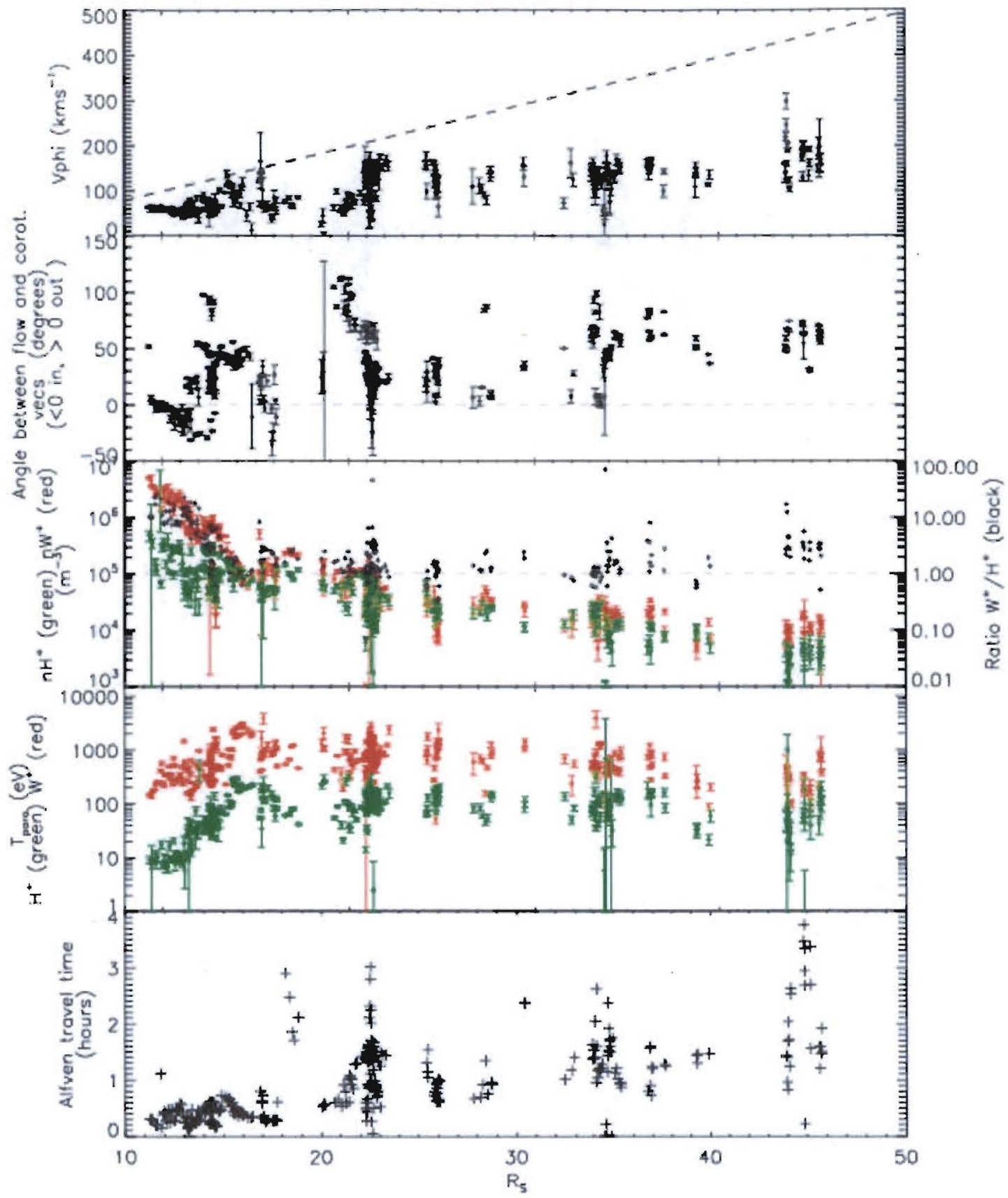




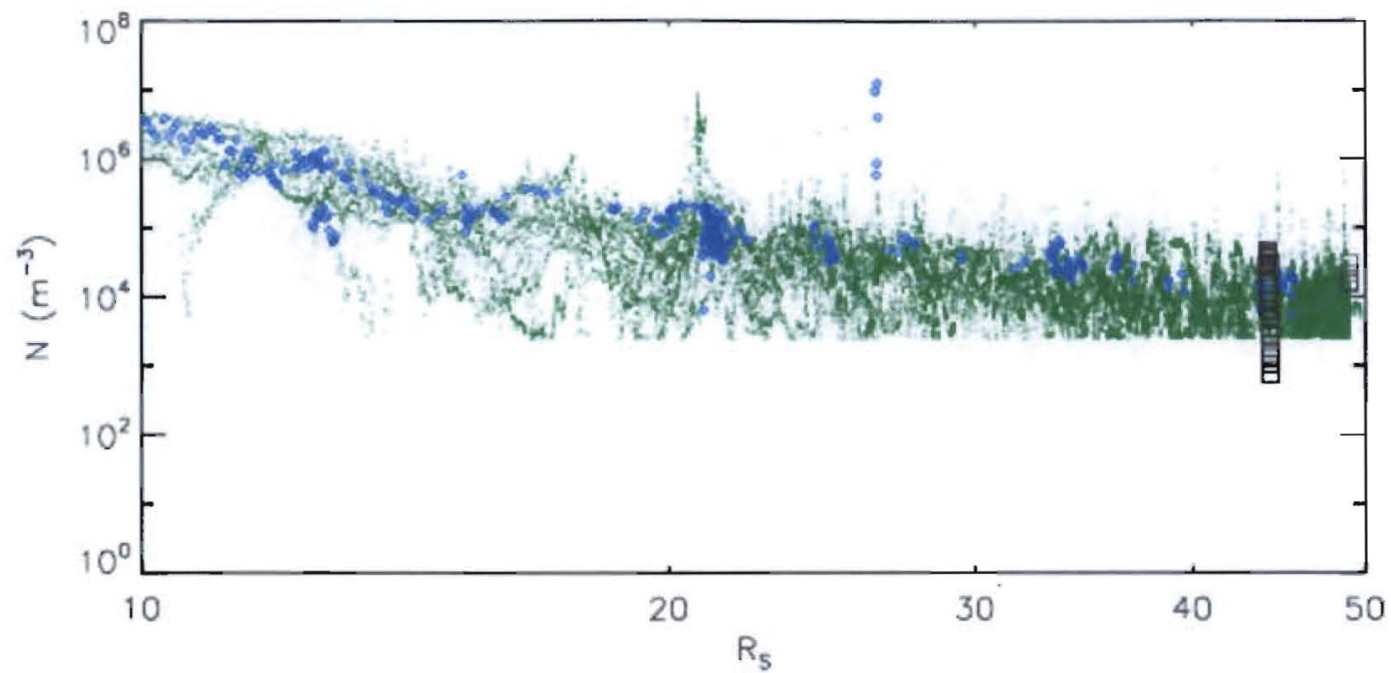




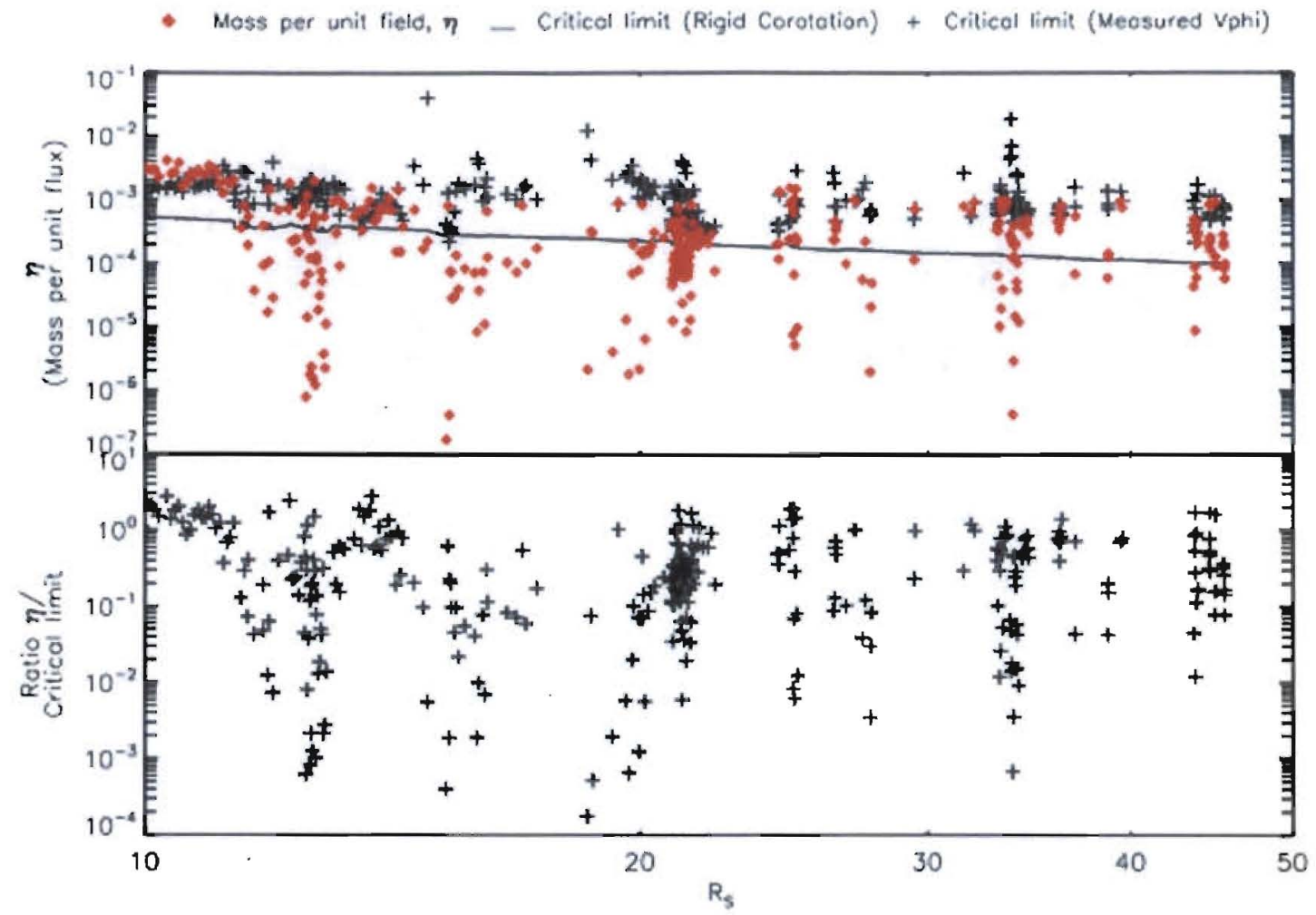

